\title{
Helium and neon photoelectron satellites at threshold
}

\author{
P. A. Heimann \\ Materials and Molecular Research Division, Lawrence Berkeley Laboratory, Department of Chemistry, \\ University of California, Berkeley, California 94720 \\ U. Becker, H. G. Kerkhoff, B. Langer, D. Szostak, and R. Wehlitz \\ Technische Universität Berlin, Institut für Strahlungs-und Kernphysik, Sekr, PN 3-2,Hardenbergstrasse 36, \\ D-1000 Berlin 12, Federal Republic of Germany \\ D. W. Lindle, T. A. Ferrett, and D. A. Shirley \\ Materials and Molecular Research Division, Lawrence Berkeley Laboratory, Department of Chemistry, \\ University of California, Berkeley, California 94720
}

(Received 12 June 1986)

\begin{abstract}
Photoionization of helium and neon to excited satellite states, $\mathrm{He}^{+} n l$ and $\mathrm{Ne}^{+} 1 s^{2} 2 s^{2} 2 p^{4} n l$, was studied with synchrotron radiation and threshold electron analysis. Photoelectron satellites have been directly measured at threshold for the first time to our knowledge. The relative satellite cross sections were determined over the kinetic energy range from 0 to $1 \mathrm{eV}$. The angular distributions were also evaluated close to threshold. Strong correlation effects were observed in two cases. For He near threshold, the angular-distribution asymmetry parameter $\beta$ is near zero for the $n=2$ satellite and is increasingly negative for the higher- $n$ satellites, in agreement with the theoretical prediction of Greene. In the threshold photoelectron spectrum of $\mathrm{Ne}$, many final states are present, some with quartet spin multiplicity and others with high- $L$ values.
\end{abstract}

\section{INTRODUCTION}

In photoelectron spectra strong "main-line" peaks are accompanied by weak satellite peaks. The satellite transitions lead to final ionic states that cannot be created simply by removing a single electron from the nominal ground-state configuration; e.g.,

$\mathrm{He}\left(1 s^{2}\left({ }^{1} S\right)\right) \rightarrow \mathrm{He}^{+}(2 s, 2 p)+e^{-}$,

or

$\mathrm{Ne}\left(1 s^{2} 2 s^{2} 2 p^{6}\left({ }^{1} S\right)\right) \rightarrow \mathrm{Ne}^{+}\left(1 s^{2} 2 s^{2} 2 p^{4}\left({ }^{1} D\right) 3 p\left({ }^{2} P^{\circ}\right)\right)+e^{-}$.

Two theoretical models which are used to describe satellite intensities are shake-up and configuration interaction (CI). According to the shake-up theory, ${ }^{1}$ the more or less abrupt change in the atomic potential that accompanies the photoelectron's departure provides a perturbation which may "shake" one of the passive electrons into an excited orbital. In this heuristic picture, the probability of reaching an excited (satellite) final state in the ion depends on the time $\Delta t$ for the hole to be created; i.e., on the kinetic energy of the photoelectron. Two limits are hypothesized. At the satellite threshold, in the adiabatic limit, the passive electrons' orbitals can relax into their final-state form during photoemission, accelerating the photoelectron. In this limit the satellite transition should have its minimal value. In the high-photon-energy, sudden limit, the satellite transition's intensity approaches a constant fraction of the main-line intensity. Thomas ${ }^{2}$ has developed a model for the transition from the adiabatic to the sudden limit. In this model the sudden limit is al- ready closely approached at a relatively low value of the satellite photoelectron's kinetic energy.

The CI model employs a multiconfiguration description of the initial and final states to calculate the satellite cross sections $\sigma$. Because of their closed-shell initial states, CI calculations have been carried out for the valence satellites of the rare gases. ${ }^{3}$ In the high-photon-energy limit, the relative satellite intensities may be calculated from the $\mathrm{CI}$ mixing coefficients. For the "final-ionic-state CI channel" in Ne $2 p$ photoemission, for example, much of the intensity is derived from the admixture of the main configuration, $\mathrm{Ne}^{+}\left|2 p^{52} P^{\circ}\right\rangle$, in the final-state wave function. By using a basis of relaxed orbitals to describe the final ionic state, the CI calculations include the shake-up contributions. To first approximation, the photon-energy dependence of the satellite to main-line intensity ratio is determined by the energy variation of the dipole matrix element between the $2 p$ and continuum orbitals. ${ }^{4}$ At a given photon energy $h v$ the satellite and main-line photoelectrons will have different kinetic energies. For the $n p^{4} n d^{2} \mathrm{~S}$ satellites in $\mathrm{Ar}, \mathrm{Kr}$, and $\mathrm{Xe}, \mathrm{Smid}$ and Hansen ${ }^{4}$ have predicted that the relative intensities will remain nearly constant with energy, decreasing slowly with increasing $h v$. However, these calculations are not expected to be accurate at low kinetic energies.

The predictions of the different theories for satellites far from the sudden limit are most rigorously tested by measuring satellites at threshold and low kinetic energies. In particular, one experiment has examined a satellite intensity through a wide range of kinetic energies down to threshold. Woodruff and Samson ${ }^{5,6}$ used synchrotron radiation to ionize $\mathrm{He}$ and the fluorescent decay from the 
excited $\mathrm{He}^{+}(n=2)$ levels to measure the $n=2$ satellite intensity down to threshold. In the low-kinetic-energy region, they observed that the satellite cross section varied slowly with energy except on autoionizing doubly excited states, where dramatic asymmetric and window profiles were observed. Since the satellite intensity was not observed to decrease at low energies, their experiment suggests that the time-dependent shake-up theory ${ }^{2}$ is not an adequate description for $\mathrm{He}$ near threshold as might be expected for a two-electron system. By having a strong electric field present or absent, Woodruff and Samson ${ }^{6}$ were also able to deconvolute the $n=2$ fluorescence into the $2 s$ and $2 p$ contributions. Their results, as well as those from photoelectron angular-distribution measurements, ${ }^{7-9}$ show the $2 s$ having a nearly constant satellite to main-line intensity ratio, while the $2 p$ to $1 s$ branching ratio decreases with increasing kinetic energy over the measured $60-\mathrm{eV}$ range. The energy-dependent behavior of $\sigma_{2 s}$ and $\sigma_{2 p}$ has been reproduced by close-coupling calculations. $10^{\circ}$

The fluorescent technique, however, is limited to the lowest-binding-energy satellite. A more general method is electron spectroscopy. Satellites in the Ar $K L L$ Auger spectrum have been studied by Armen et al. ${ }^{11}$ In qualitative agreement with the predictions of the time-dependent shake-up theory, the core-level Auger satellite is weaker near threshold, increasing to a constant value at higher photon energies. Experiments on the valence photoelectron satellites of $\mathrm{Ne},{ }^{12,13} \mathrm{Ar},{ }^{14}$ and $\mathrm{Xe}$ (Ref. 15) have revealed a variety of intensity versus energy dependences: increasing, nearly constant, and decreasing. Quite different $\sigma(h v)$ behavior has been seen for satellites whose main final-state configurations have the same excited orbital. $^{12,13}$ Shake-up and initial- and final-ionic-state CI alone are unable to explain the observed variety of $\sigma(h v)$ behavior. Mechanisms not included in these theories are autoionization from doubly excited states, studied in $\mathrm{He}$ (Refs. 5-8) and $\mathrm{Ne},{ }^{13}$ and continuum-state $\mathrm{CI}$, which has been observed in the He $2 p$ energy dependence. ${ }^{6-9} \mathrm{Au}$ toionization into satellite channels and continuum-state CI have together been referred to as interchannel coupling.

These additional processes should be strongest at threshold. Unfortunately, electron spectrometers typically have increasing difficulties with background and transmission as the electron energy decreases below a few eV. However, one class-threshold electron analyzersare specifically designed to study photoionization processes at threshold by using a small electric field for extracting slow electrons. ${ }^{16-18}$ For $0-\mathrm{eV}$ electrons this technique provides high sensitivity and high resolution. A threshold electron analyzer has been constructed which uses timeof-flight analysis, similar to the approach used by Baer et al. ${ }^{16}$ Because of its longer flight distance, the resolution of our analyzer degrades more slowly with increasing kinetic energy, facilitating the measurement of satellite intensities at small kinetic energies. Combining the threshold electron analyzer with a rotatable chamber allows satellite angular distributions to be determined near threshold, as well. In this paper we report studies of correlation satellites in the photoelectron spectra of $\mathrm{He}^{+}$ and $\mathrm{Ne}^{+}$, determined near threshold by this spectrometer. Experimental procedures are described in Sec. II. In Sec. III we report and discuss the cross sections and asymmetry parameters of the $\mathrm{He}^{+}$satellites. Neon satellite cross sections and asymmetry parameters are presented and discussed in Sec. IV. Finally, Sec. V contains the conclusions.

\section{EXPERIMENTAL}

Figure 1(a) shows the threshold electron analyzer, which is cylindrically symmetrical. At the interaction region the synchrotron radiation beam crosses through an effusive gas jet. Electrons ejected from the gaseous atoms pass through three regions: the extraction region $(10 \mathrm{~mm}$ long), the acceleration region $(18 \mathrm{~mm})$, and the drift region $(262 \mathrm{~mm})$. With the voltages plotted in Fig. 1(b), zero-kinetic-energy electrons have a flight time of $150 \mathrm{~ns}$. Figure 2 shows a photoelectron spectrum of $\mathrm{He}$ with the photon energy $1 \mathrm{eV}$ above the $n=2$ satellite threshold. Two peaks result from the $n=2$ satellite transition because of photoelectrons initially moving toward (shorter time) and away from (longer time) the detector.

The small electric field across the interaction region bends the trajectories of low-kinetic-energy electrons toward the detector. For very slow electrons, in our case 0-30 meV, all emission directions are accepted. The transmission function shown in Fig. 2 was calculated by considering a point source and the initial ejection angle for an electron to hit the edge of the detector. The transmission decreases steeply to an acceptance half-angle $(\delta)$ of $10^{\circ}$ at $1 \mathrm{eV}$. However, the $1-\mathrm{eV}$ angular acceptance is still much larger than that of an angle-resolved photoelectron analyzer. For example, the time-of-flight electron spectrometers of White et al. ${ }^{19}$ have a collection half-angle $\delta$ of $3^{\circ}$.

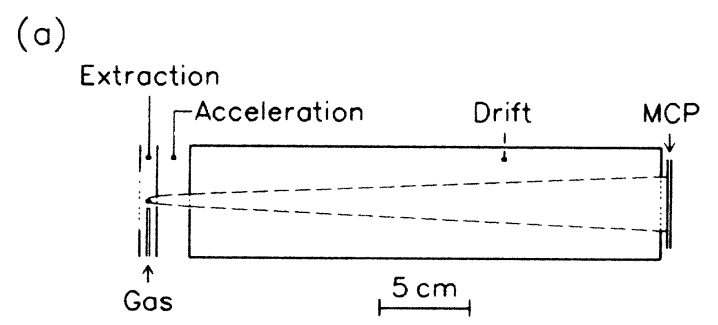

(b)

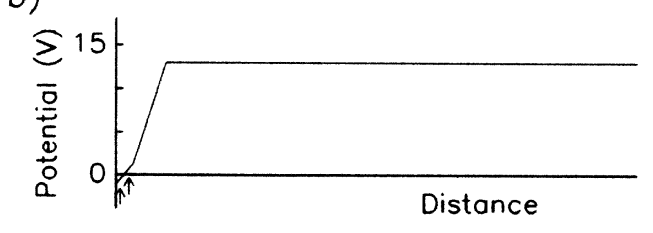

FIG. 1. (a) A schematic diagram of the threshold electron analyzer drawn to scale. Two electron trajectories are represented by long dashed lines. (b) The varying potential $(V)$ seen by the photoelectron as it leaves the ionization region and moves toward the microchannel plate detector [scale same as in (a)]. The arrows point to initial positions for an electron on either side of the ionization region. 


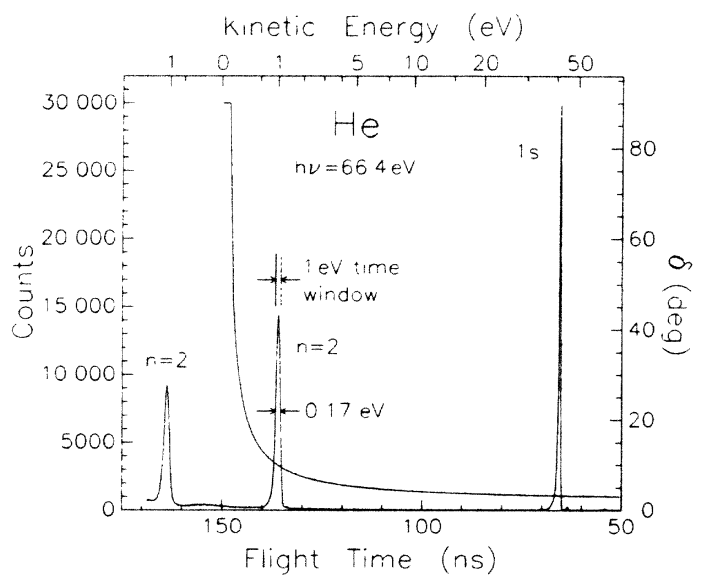

FIG. 2. A time spectrum of He taken near the $n=2$ satellite threshold in $500 \mathrm{~s}$ (left axis). Also shown is the calculated transmission function expressed as the acceptance half-angle $\delta$ (right axis).

A threshold time-of-flight analyzer, like a time-offlight mass spectrometer, achieves high resolution by satisfying the space focusing condition. ${ }^{20}$ As shown in Fig. 1(b), an electron from the side of the interaction region further from the detector is accelerated more than one from the near side. With the proper choice of distances and voltages, both electrons arrive at the detector in the same flight time. One-dimensional calculations which consider a 5-mm-wide interaction region show that the resulting time width can be less than $1 \mathrm{~ns}$ for electrons with kinetic energies from 0 to $10 \mathrm{eV}$. Ray tracing for $0.1-$ and $1-\mathrm{eV}$ electrons predicts that the time width from the different initial emission directions should also be less than 1 ns. These calculations are confirmed by the observed full width at half maximum of $1.3 \mathrm{~ns}$ for the $1-\mathrm{eV}$ peak in Fig. 2. The monochromator bandpass contributes significantly to this width.

We now describe some other aspects of the design. A moveable hypodermic needle serves as the gas inlet while preserving the uniformity of the electric field. The analyzer apertures are wide in order to accept electrons from a large interaction region. The first and smallest aperture has a diameter of $6 \mathrm{~mm}$. The apertures are covered with $\mathrm{Cu}$ grids, 20 lines per inch. For the shielding of magnetic fields, there are two cylinders of $\mu$ metal around the analyzer and one cylinder at the vacuum chamber wall. The drift region and detector are differentially pumped by a 50-1/s turbo pump. The electron detector, consisting of a pair of microchannel plates and a conical anode, is identical to that of White et al. ${ }^{19}$ To minimize unwanted secondary electrons, all surfaces seen by photoelectrons in flight are coated with graphite. In order to further reduce the background, an aperture is positioned upstream of the analyzer to prevent scattered light from hitting any analyzer surfaces.

The time-of-flight signal processing is done in the usual way ${ }^{19}$ using a time-to-amplitude converter (TAC), which receives the electron pulse as "start" and the light pulse as "stop." Scans are done by varying the photon energy and having a multi-input scalar collect (i) the counts falling into one or two time (kinetic energy) windows and (ii) the output from the photon flux monitor, a sodium salicylate scintillator. For normalization the electron counts are divided by the photon flux. Within a scan the electrons are all at a given kinetic energy. Therefore, in a scan the normalized intensities are proportional to the differential cross section $d \sigma(h v, \theta) / d \Omega$, with no analyzer transmission correction needed. The sample pressure was held constant by a variable leak valve. The ambient pressure of the chamber was about $3 \times 10^{-5}$ mbar during both the $\mathrm{He}$ and $\mathrm{Ne}$ experiments.

The experiments were performed at the 5.6-m toroidal grating monochromator (TGM) at the Hamburger Synchrotronstrahlungslabor (HASYLAB). Some preliminary scans were carried out using a grazing incidence "Grasshopper" monochromator at the Stanford Synchrotron Radiation Laboratory (SSRL). For He a 1500 lines per $\mathrm{mm}$ grating with no filter was used. For $\mathrm{Ne}$ a 750 lines per mm grating was employed with a $1500-\AA$ Al filter to reduce the contribution of higher-order light. Residual higher-order light produces a continuous background because the higher-order components are above the threshold for double ionization. In addition, the measured light intensity must be corrected in order to represent the first-order component alone. The typical resolution of the TGM monochromator was $0.1 \mathrm{eV}$ for the $0-\mathrm{eV}$ scans and $0.2 \mathrm{eV}$ for the higher-kinetic-energy scans.

Figure 3 shows a $0-\mathrm{eV}$ scan with the $\mathrm{He}$ satellites $n=2-8$ clearly seen with smoothly decreasing intensity. At $65.4 \mathrm{eV}$ photon energy, the combined analyzer and monochromator resolution is $0.15 \mathrm{eV}$, of which the monochromator contributes an estimated $0.08 \mathrm{eV}$. The $n=8$ satellite has a cross section of only $0.8 \mathrm{~kb}$, which demonstrates the high sensitivity of the threshold technique. The higher satellites are seen as an unresolved step which ends at the $\mathrm{He}^{2+}$ threshold. The $0-\mathrm{eV}$ signal rises above the $\mathrm{He}^{2+}$ threshold as the double-ionization probability increases.

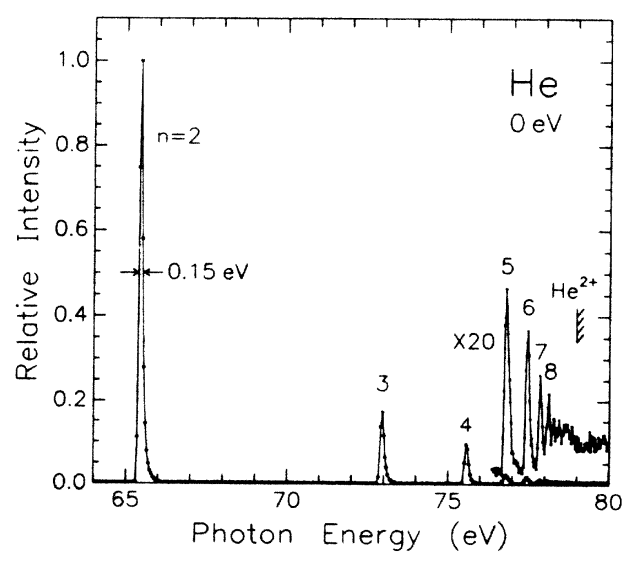

FIG. 3. Threshold photoelectron scan of the He satellites. $n$ is the principal quantum number of the remaining electron in $\mathrm{He}^{+}$. The count rate at the maximum of the $n=2$ satellite peak is 9700 counts per second. The line simply connects the data points. 
The peaks in a constant kinetic energy scan are not symmetric. For a $0-\mathrm{eV}$ scan the low- $h v$ side contains only the monochromator width, while the high- $h v$ side is affected by both the monochromator and analyzer resolutions. As a result, the peaks were fitted using an asymmetric Gaussian function, for which the left and right sides can have different widths.

The satellite cross sections were calculated from intensity ratios relative to the $n=2$ satellite for He and to the $2 s$ main line for $\mathrm{Ne}$. The $\mathrm{He} n=2$ satellite cross section has been measured by Woodruff and Samson ${ }^{5}$ to be $0.10(1)$ $\mathrm{Mb}$ from 0 to $1 \mathrm{eV}$ kinetic energy. For $\mathrm{Ne}, \sigma_{2 s}$ has only been determined down to $2.5 \mathrm{eV} .^{21}$ This lowest kinetic energy value $\sigma_{2 s}=0.21(4) \mathrm{Mb}$ (assumed to include satellite 13) was extrapolated linearly to $\sigma_{2 s}=0.17 \mathrm{Mb}$ at $0 \mathrm{eV}$. The reported errors for the cross sections represent the uncertainties of the fits, increased if two scans do not agree within errors. These error estimates are as large as, or larger than, the statistical uncertainty.

The analyzer was mounted in a chamber which could be rotated about the photon beam. ${ }^{22}$ In order to determine the angular-distribution asymmetry parameter $\beta$, we carried out 0.5 and $1 \mathrm{eV}$ scans at three angles: $\theta=0^{\circ}, 48^{\circ}$ or $55^{\circ}$, and $90^{\circ}$. Here, $\theta$ is the angle between the photon polarization direction and the electron emission direction. In the dipole approximation with a randomly oriented sample, the angular distribution of photoelectrons follows the equation given by Yang, ${ }^{23}$

$$
\frac{d \sigma(h v, \theta)}{d \Omega}=\frac{\sigma}{4 \pi}\left[1+\beta(h v) P_{2}(\cos \theta)\right],
$$

where $P_{2}$ is the second Legendre polynomial. In analyzing the angular distribution results, two sizable corrections must be considered. First, the incomplete linear polarization of the light must be included. Second, the large acceptance angle $(\delta)$ of the analyzer must be considered. The resulting equation for the photoelectron angular distribution is

$$
\begin{array}{r}
\frac{1}{\sigma} \int d \sigma=\frac{1}{4}[ \\
2-2 \cos \delta+\frac{1}{4}\left(1-P_{1}\right) \beta \cos \delta \sin ^{2} \delta \\
\left.+P_{1} \beta \cos \delta \sin ^{2} \delta P_{2}(\cos (\theta-\lambda))\right] .
\end{array}
$$

The first two terms represent the angle-independent part, which becomes monotonically larger as $\delta$ increases. The last two terms come from the angle-dependent portion of Eq. (1).

The polarization calibration was performed by taking photoelectron spectra of $\mathrm{Ne}$ at several angles and by using the known $\beta$ values of the $2 s$ and $2 p$ lines. The measured values for the $\mathrm{He}$ experiment $(60-80-\mathrm{eV}$ photon energy) were $P_{1}=0.89(2), \lambda=-7(2)^{\circ}$ and for the Ne experiment $(50-60 \mathrm{eV}), P_{1}=0.73(3), \lambda=-10(1)^{\circ}$, where $P_{1}$ is the first Stokes parameter $\left(I_{\|}-I_{\perp}\right) /\left(I_{\|}+I_{\perp}\right)$ and $\lambda$ the angle between the major axis of the polarization ellipse and the horizontal plane of the DORIS storage ring. Measuring the intensity ratio of $\mathrm{He}(n=2)$ at low kinetic energy and $\mathrm{He} 1 s$ at high kinetic energy while assuming the calculated $\delta$ at high energy gives the following results: $\delta=15^{\circ}$ at $0.5 \mathrm{eV}$ and $\delta=11^{\circ}$ at $1 \mathrm{eV}$. These $\delta$ values, which represent average acceptance angles over the source volume, are quite similar to those calculated for a point source at 0.5 and $1.0 \mathrm{eV}$. The analyzer sensitivity was very angle dependent in part because of the crescentshaped photon beam spot, $5 \mathrm{~mm}$ broad. As a result, the sensitivity to $\theta$ had to be calibrated against a transition of known $\beta$.

\section{HELIUM}

The cross sections and asymmetry parameters will be discussed separately below.

\section{A. Helium cross sections $(\sigma)$}

Table I shows the observed He satellite binding energies together with the calculated energies of a singly charged $\mathbf{H}$-like ion. Each satellite contains an unresolved summation of the possible values of the orbital angular momentum $l$. Table I displays in its last column the observed $\sigma$ values at $0 \mathrm{eV}$. At threshold the satellite $\sigma$ values decrease approximately as the mean separation of energy levels adjacent to $E_{n}, \Delta E(n)=8 I_{\mathrm{H}} n /\left(n^{2}-1\right)^{2}$, with $I_{\mathrm{H}}$ being the ionization potential of $\mathrm{H}$. Fano and Cooper ${ }^{25}$ state that for a given Rydberg series, the excited orbitals are nearly identical at small $r$ except for a normalization factor, for which they use the energy spacing. Qualitatively, the variation of the satellite $\sigma$ values with $n$ reflects the expansion of the Rydberg orbitals.

The satellite $\sigma$ values from 0 to $1 \mathrm{eV}$ above threshold are shown in Fig. 4. The $\sigma$ values in both the table and the figure have been corrected for the contribution of higher-order light, which decreases nearly linearly from $31 \%$ at $65 \mathrm{eV}$ to $12 \%$ at $80 \mathrm{eV}$ photon energy. There are no calculated $\sigma$ values available for comparison. A recent photoelectron measurement at higher kinetic energies ${ }^{26}$ shows $\sigma_{n=3}$ and $\sigma_{n=4}$ having lower values than at thresh-

TABLE I. The He satellite binding energies and threshold cross sections.

\begin{tabular}{lccl}
\hline \hline \multicolumn{1}{c}{ Peak } & $\begin{array}{c}\text { Binding } \\
\text { energy }(\mathrm{eV})\end{array}$ & $\begin{array}{c}\text { Calculated } \\
\text { energy }(\mathrm{eV})^{\mathrm{a}}\end{array}$ & \multicolumn{1}{c}{$\sigma(\mathrm{Mb})$} \\
\hline $1 s$ & & 24.567 & $7.6(2)^{\mathrm{b}}$ \\
$n=2$ & 65.44 & 65.377 & $0.10(1)^{\mathrm{c}}$ \\
$n=3$ & 72.95 & 72.934 & $0.0159(5)$ \\
$n=4$ & 75.57 & 75.579 & $0.0080(2)$ \\
$n=5$ & 76.80 & 76.803 & $0.0022(1)$ \\
$n=6$ & 77.46 & 77.469 & $0.0015(1)$ \\
$n=7$ & 77.86 & 77.870 & $0.00091(7)$ \\
$n=8$ & 78.12 & 78.130 & $0.00077(7)$ \\
$n=9$ & 78.30 & 78.308 & $0.00043(7)$ \\
$n=10$ & 78.46 & 78.436 & $0.00037(6)$ \\
Sat. total & & & 0.13 \\
\hline \hline
\end{tabular}

${ }^{\text {a From }} E_{n}=E_{\infty}-I_{\mathrm{H}}\left(Z^{2} / n^{2}\right)$, where $I_{\mathrm{H}}$ is the ionization energy for $\mathbf{H}$.

${ }^{\text {b Reference } 24 .}$

${ }^{\text {c}}$ Reference 5 . 


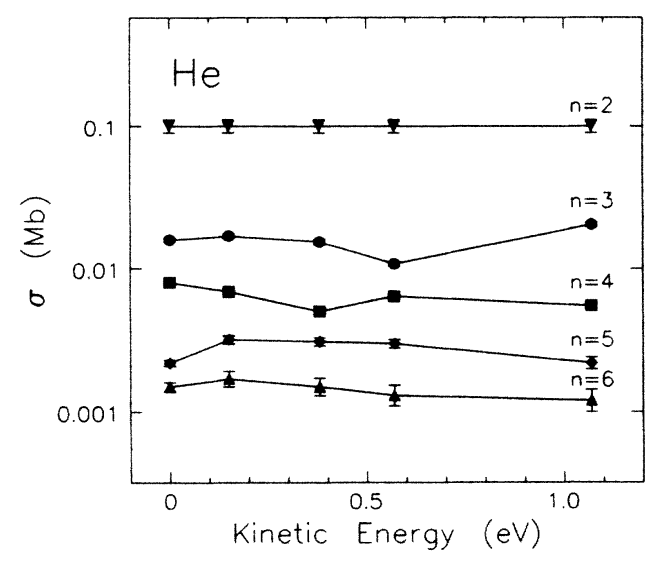

FIG. 4. He satellite cross sections at low kinetic energies shown in a semilog plot. The $n=2$ satellite $\sigma$ values are from Woodruff and Samson (Ref. 5).

old, with the intensities decreasing faster than the $1 s$ main line. The satellites as a whole show relatively constant behavior over the kinetic energy range from 0 to $1 \mathrm{eV}$. Continuum-state $\mathrm{CI}$ has been shown both theoretically ${ }^{10}$ and experimentally ${ }^{6-9}$ to strengthen the $2 p$ satellite as the kinetic energy decreases. It should affect the higher- $n$ satellites in a similar manner. However, the intensity variations caused by continuum-state CI, as observed for He $2 p$, occur over wide ranges of kinetic energy. The resulting intensity change from 0 to $1 \mathrm{eV}$ should be quite small. The changes in intensity which are observed probably result from autoionization from doubly excited states. A number of autoionizing resonances $K_{n}$ have been reported: ${ }^{6} 2_{4}$ at $73.66 \mathrm{eV}, 0_{4}$ at $74.15 \mathrm{eV}, 3_{5}$ at 75.54 $\mathrm{eV}, 3_{6}$ at $76.10 \mathrm{eV}$, and $3_{7}$ at $76.30 \mathrm{eV}$, all of which could contribute to variations in Fig. 4. A more detailed study is required to determine the effect of individual resonances on each satellite.

\section{B. Helium asymmetry parameters $(\beta)$}

Figure 5 shows the asymmetry parameters of the $\mathrm{He}$ satellites $n=2-6$ measured at kinetic energies near 0.5 and $1.0 \mathrm{eV}$. The relative analyzer efficiency $E(\theta)$ was calibrated by using known $\beta$ values for one transition. For $0^{\circ} E$ was alternatively inferred from $\beta_{1 s}=2$ and from $\beta_{n=2}=-0.10(12){ }^{7} E\left(55^{\circ}\right)$ was set arbitrarily to 1 , while $\beta_{n=2}$ was again used to evaluate $E\left(90^{\circ}\right)$. The error bars for $\beta$ include both statistical errors and systematic errors from the efficiency calibration, estimated to be 0.1 . The $\beta_{n=2}$ values reported result from the $\beta_{1 s}$ calibration and agree with the earlier measurements. ${ }^{7-9}$ Lindle et al. ${ }^{26}$ determined $\beta$ for the satellites $n=3,4$, and 5 at some higher kinetic energies. Their lowest kinetic energy $\beta$ values, at 3-4 eV, agree with the present results, while at higher energies $\beta_{n=3}$ and $\beta_{n=4}$ are observed to increase.

Greene $^{27}$ has calculated the near-threshold asymmetry parameters for $\mathrm{He}$. His theoretical approach starts from Herrick's $^{28}|K T\rangle$ basis, developed for doubly excited

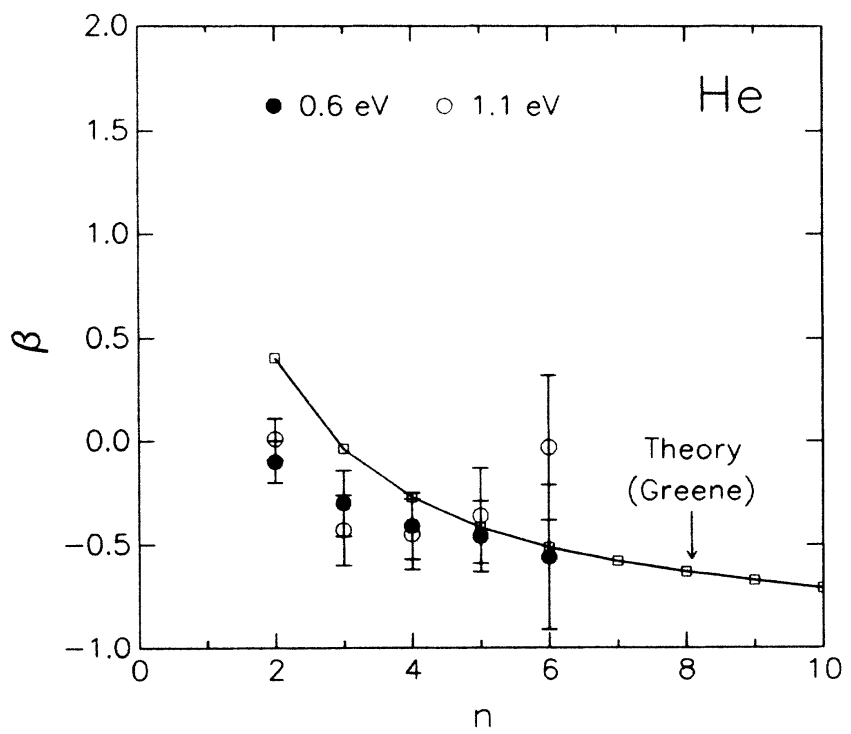

FIG. 5. Asymmetry parameter $(\beta)$ of the $\mathrm{He}$ satellites displayed as a function of the principal quantum number $n$ of the $\mathrm{He}^{+}$excited electron. The circles represent two sets of measurements at $0.6 \mathrm{eV}$ (solid circles) and at $1.1 \mathrm{eV}$ (open circles) kinetic energies. The open squares show the calculation of Greene (Ref. 27). They are connected by a line only for clarity.

states. In this basis the dipole operator $r_{1} \cos \theta_{12} / r_{2}^{2}$ is approximately diagonal. Here $r_{1}$ is the radial distance of the bound electron, $r_{2}$ the radial distance of the photoelectron and $\theta_{12}$ the angle between $\hat{\boldsymbol{r}}_{1}$ and $\hat{\boldsymbol{r}}_{2}$. Larger values of $K$ and $T$ correspond to more correlated angular motion of the two electrons, which tends to keep them on opposite sides of the nucleus. For both near-threshold satellites and doubly excited states, the dipole operator has dominant importance for the angular wave functions. In both these cases angular correlations are influential. For He with a ${ }^{1} P^{\circ}$ final state, the $T=1$ channel is the lowest energy channel at high $r_{2}$. For He with $T=1$, the preferential photoelectron direction is parallel or antiparallel to $\mathbf{L}=\mathbf{j}_{\gamma}$, and $\beta \sim-1$, where $\mathbf{L}$ is the total orbital angular momentum and $\mathbf{j}_{\gamma}$ is the angular momentum of the photon. The results calculated by Greene are displayed in Fig. 5 as open squares, connected by a solid line for clarity. Both the experiment and theory show $\beta$ decreasing with increasing $n$. The generally good agreement between experiment and theory implies that the factors affecting $\beta$ are reasonably well understood and are included in Greene's theory. This is the major result of the comparison. The decreasing trend in $\beta$ also confirms the expectation that the $K, T$ classification becomes more exact as $n$ increases. The agreement between the experiment and the calculation of Greene also improves with increasing $n$. Finally, Greene only claimed accuracy for his calculation for photoelectron energies between 0 and a fraction of an $\mathrm{eV}$. However, the small change in $\beta$ from 0.6 to $1.1 \mathrm{eV}$, as well as the agreement with the measured $\beta$ values at $3-4 \mathrm{eV},{ }^{26}$ suggests that the theory and the angular correlations which it describes may in fact apply to a wider kinetic energy range than expected. 
An alternative basis set for the $\mathrm{He}$ final state is $\left|n l_{1} l_{2} ; L \pi\right\rangle$, where $n$ and $l_{1}$ refer to the bound electron and $l_{2}$ to the photoelectron. In this description each satellite $n$ has a set of $l_{1}$ unresolved components, with $\beta_{n}$ determined by

$$
\beta_{n}=\sum_{l_{1}} \sigma_{l_{1}} \beta_{l_{1}} / \sum_{l_{1}} \sigma_{l_{1}}
$$

The extraction field was much too weak to produce significant $l_{1}$ mixing. For the $n=2 \mathrm{He}$ satellite where $l_{1}$ may be $s$ or $p$; Jacobs and Burke ${ }^{10}$ and Chang $^{29}$ have calculated $\beta_{2 p}$ which decreases with decreasing kinetic energy until $\beta_{2 p}=-0.85$ at $1.3 \mathrm{eV}$ (from Jacobs and Burke). All $\beta_{n s}$ must be 2. From $\beta_{2 s}$ and $\beta_{2 p}$ along with our experimental $\beta_{n=2}$, one infers $\sigma_{2 p} / \sigma_{2 s}=2.7(5)$ at $0.6 \mathrm{eV}$ and 2.3(4) at $1.1 \mathrm{eV}$. Like $\beta_{n=2}$, these ratios agree with earlier measurements. ${ }^{6,8}$ For the higher- $n$ satellites larger $l_{1}$ and $l_{2}$ can contribute. Since only $\beta_{n s}$ is known a priori, and only $\beta_{2 p}$ is available from calculations, the different $l_{1}$ components are undetermined for $n>2$. However, the observed negative $\beta$ values imply that the $n s$ states cannot be dominant constituents in the $n>2$ satellites. Figure 6(a) shows Greene's ${ }^{30}$ threshold predictions for the fractional contribution of the different $l_{1}$ values in a given $n$ satellite peak. As $n$ increases, the distribution over $l_{1}$ shifts toward higher $l_{1}$. We shall return to Fig. $6(\mathrm{~b})$ at the end of Sec. IV.

\section{NEON}

\section{A. Neon cross sections $(\sigma)$}

Figure 7 shows the $0-\mathrm{eV}$ scan of the $\mathrm{Ne}^{+}(2 s)$ and $\mathrm{Ne}^{+}\left(1 s^{2} 2 s^{2} 2 p^{4} n l\right)$ satellites. At $48.5 \mathrm{eV}$, the combined analyzer and monochromator resolution is $0.14 \mathrm{eV}$, of which the monochromator contributes an estimated 0.11 eV. Table II contains assignments of the observed peaks

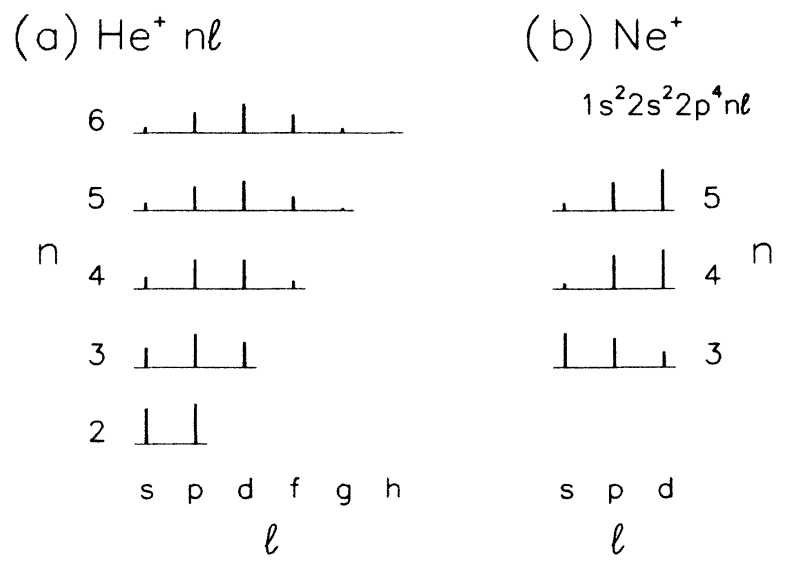

FIG. 6. The relative $n l$ distribution of the excited electron in the threshold satellite spectrum: (a) He calculation (Ref. 30), (b) $\mathrm{Ne}$ experiment (this work). For each $n$ the $l$ segments sum to a unit length. Included in the $\mathrm{Ne} 4 d$ and $5 d$ fractions are unresolved higher- $l$ components. using the optical energies of Persson. ${ }^{31}$ The table also includes peaks observed at higher photon energy than shown in Fig. 7. This rather long list is not meant to be complete, but instead includes the states which are necessary to obtain a good fit. Additional states could contri-

TABLE II. Neon valence satellite binding energies from the present work together with the energies of Persson (Ref. 31) from emission spectroscopy. All the assigned satellite final states include a $1 s^{2} 2 s^{2} 2 p^{4}$ core. The last column contains the satellite cross sections at threshold measured by us.

\begin{tabular}{|c|c|c|c|c|}
\hline Peak & $\begin{array}{c}\text { Binding } \\
\text { energy }(\mathrm{eV})\end{array}$ & Assignment & $\begin{array}{c}\text { Optical } \\
\text { energy }(e V)\end{array}$ & $\sigma(\mathrm{Mb})$ \\
\hline $2 p$ & & & 21.57 & $6.2(2)^{\mathrm{a}}$ \\
\hline $2 s$ & & & 48.48 & $0.16^{b}$ \\
\hline 13 & 48.77 & $\left({ }^{3} P\right) 3 s^{4} P$ & 48.77 & $0.013(3)$ \\
\hline 12 & 49.36 & $\left({ }^{3} P\right) 3 s^{2} P$ & 49.37 & $0.019(3)$ \\
\hline 11 & 52.07 & $\left({ }^{1} D\right) 3 s^{2} D$ & 52.11 & $0.063(7)$ \\
\hline $11 \mathrm{a}$ & 52.43 & $\left({ }^{3} P\right) 3 p^{4} D^{\circ}$ & 52.49 & $0.007(2)$ \\
\hline \multirow[t]{2}{*}{$10 \mathrm{a}$} & 52.72 & $\left({ }^{3} P\right) 3 p^{2} D^{\circ}$ & 52.71 & $0.007(2)$ \\
\hline & & $\left({ }^{3} P\right) 3 p^{2} S^{\circ}$ & 52.91 & \\
\hline 10 & 53.07 & $\left({ }^{3} P\right) 3 p^{2} P^{\circ}$ & 53.08 & $0.027(3)$ \\
\hline $7 a$ & 55.58 & $\left({ }^{1} D\right) 3 p^{2} F^{\circ}$ & 55.59 & $0.009(2)$ \\
\hline \multirow[t]{2}{*}{7} & 55.81 & $\left({ }^{1} D\right) 3 p^{2} P^{\circ}$ & 55.83 & $0.024(3)$ \\
\hline & & ${ }^{2} D^{\circ}$ & 55.95 & \\
\hline \multirow[t]{4}{*}{6} & 56.33 & $\left({ }^{3} P\right) 3 d{ }^{4} D$ & 56.19 & $0.020(3)$ \\
\hline & & ${ }^{2} D$ & 56.32 & \\
\hline & & ${ }^{2} F$ or ${ }^{4} P$ & 56.39 or 56.41 & \\
\hline & & ${ }^{2} P$ & 56.48 & \\
\hline $6 a$ & 56.66 & $\left({ }^{3} P\right) 4 s^{2} P$ & 56.72 & $0.005(2)$ \\
\hline 5 & 57.68 & $\left({ }^{3} P\right) 4 p^{4} D^{\circ}$ & 57.71 & $0.004(1)$ \\
\hline & & + other terms & & \\
\hline 4 & 57.99 & $\left({ }^{3} P\right) 4 p^{2} P^{\circ}$ & 58.04 & $0.013(2)$ \\
\hline \multirow[t]{3}{*}{$3 b$} & 59.04 & $\left({ }^{3} P\right) 4 d{ }^{4} D$ & 59.00 & $0.016(2)$ \\
\hline & & ${ }^{2} D$ & 59.05 & \\
\hline & & ${ }^{2} P$ & 59.15 & \\
\hline \multirow[t]{3}{*}{3} & 59.40 & $\left({ }^{3} P\right) 5 s^{2} P$ & 59.27 & $0.029(3)$ \\
\hline & & $\left({ }^{1} S\right) 3 p^{2} P^{\circ}$ & 59.43 & \\
\hline & & $\left({ }^{1} D\right) 3 d^{2} D$ & 59.54 & \\
\hline \multirow[t]{2}{*}{$3 \mathbf{a}$} & 59.67 & $\left({ }^{3} P\right) 5 p^{4} D^{\circ}$ & 59.69 & $0.009(2)$ \\
\hline & & ${ }^{2} P^{\circ}$ & 59.85 & \\
\hline $2 a$ & 60.31 & $\left({ }^{3} P\right) 5 d$ & 60.3 & $0.008(2)$ \\
\hline 2 & 60.96 & $\left({ }^{1} D\right) 4 p^{2} P^{\circ}$ & 60.96 & $0.009(2)$ \\
\hline $1 b$ & 61.34 & & & $0.003(1)$ \\
\hline $1 \mathrm{a}$ & 61.64 & & & $0.003(1)$ \\
\hline 1 & 62.22 & $\left({ }^{1} D\right) 4 d$ & 62.2 & $0.018(3)$ \\
\hline $\mathrm{Ob}$ & 62.7 & $\left({ }^{1} D\right) 5 p$ & & $0.001(1)$ \\
\hline $0 a$ & 63.1 & $\left({ }^{1} S\right) 3 d^{2} D$ & & $0.003(1)$ \\
\hline 0 & 63.4 & $\left({ }^{1} D\right) 5 d$ & & $0.008(1)$ \\
\hline-1 & 64.0 & & & $0.003(2)$ \\
\hline-2 & 64.4 & $\left({ }^{1} S\right) 4 p^{2} P^{\circ}$ & & $0.003(2)$ \\
\hline Sat. total & & & & 0.32 \\
\hline
\end{tabular}

${ }^{\text {a Reference } 24 .}$

${ }^{\text {b } R e f e r e n c e ~} 21$. 


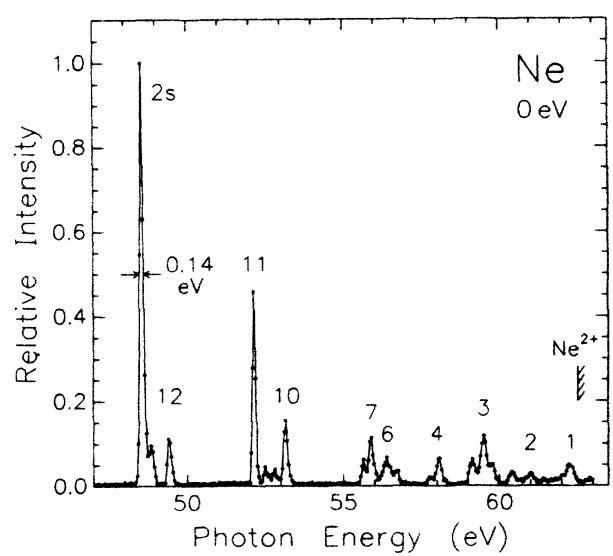

FIG. 7. Threshold photoelectron scan of the Ne $2 s$ main line and valence satellites. The numbers labeling the satellite peaks follow the notation of Wuilleumier and Krause (Ref. 32). For clarity the additional peaks are not labeled, but appear in Table II. As in Fig. 3 the line connects the points.

bute, for example, $\left({ }^{1} S\right) 3 s^{2} S$ at $55.87 \mathrm{eV}$ and $\left({ }^{1} D\right) 3 d^{2} S$ at $59.51 \mathrm{eV}$. Above $59 \mathrm{eV}$, as the energy approaches the double-ionization thresholds, the assignments become more uncertain because the density of final states increases greatly. Both the $d$ and $f$ Rydberg orbitals have small quantum defects because of large centrifugal barriers. The energy spacing between $n d$ and $n f$ states is therefore quite small. As a result, the satellite peaks assigned to $4 d$ and $5 d$ states in Table II probably contain $n f$ contributions.

The spectral scan in Fig. 7 contains many more peaks and its assignment contains many more final states than have been previously observed in Ne spectra. ${ }^{12,13,32}$ Term symbols of high orbital angular momentum, e.g., ${ }^{2} F^{\circ}$, are present. The ionic term symbols populated in the shakeup and final-ionic-state CI models, ${ }^{2} P^{\circ}$ and ${ }^{2} S$, are in the minority. Several quartet states are observed contrary to the spin selection rule $\Delta S=0$. The satellite spectrum, at least at threshold, is seen to be very complex. The additional final states may or may not contribute to the satellite spectrum at higher $h v$. Comparison with photoelectron spectra ${ }^{12}$ is inconclusive because of their lower resolution of $0.4 \mathrm{eV}$ and higher background.

An analogy with double ionization suggests that the satellite spectrum at threshold might be different from that observed at higher energies. Fano ${ }^{33}$ has predicted the excitation of high orbital angular momentum states near the double-photoionization threshold (below and above). With two electrons moving slowly from the ion core, the angular correlation predicted by the Wannier theory ${ }^{34}$ restricts the electrons to two narrow cones in opposite directions. The narrow spatial angles imply that the electrons may attain high values of $l$. For satellites at threshold, the situation is similar: the photoelectron and excited electrons are traveling away from the nucleus with small kinetic energy. The satellite may be thought of as a failed double ionization where one electron falls behind the other and is captured. In an analogous way, the spectator decay of an inner-shell resonance has been considered as an extreme case of post-collision interaction. ${ }^{35}$ The observed quartet and high- $L$ satellite states in $\mathrm{Ne}$ may result from the strong coupling between the excited electron and photoelectron. The orientation of the core's spin and orbital angular momentum exerts a weakened influence on the excited electron. However, certain term symbols for the complete ion are still favored such as ${ }^{2} P^{\circ}$ and ${ }^{2} D$.

Autoionization from doubly excited states could explain an additional satellite being present at threshold. As seen by Woodruff and Samson ${ }^{5,6}$ in He and Becker et al. ${ }^{13}$ in $\mathrm{Ne}$, this mechanism is especially important at low kinetic energies because there exists a series of doubly excited levels below each satellite threshold. The measurements in $\mathrm{Ne}$ showed in particular that satellite $12\left({ }^{3} P\right) 3 s^{2} P$ has vanishing intensity except on doubly excited resonances. The influential doubly excited states have either the same core term symbol $\left({ }^{3} P\right)$ or the same excited orbital $(3 s)$ as satellite 12. The absorption experiment of Codling et $a l .{ }^{36}$ places resonance $\left({ }^{3} P\right) 3 p\left({ }^{2} P\right) 4 d$ at $52.112 \mathrm{eV}$, $\left({ }^{3} P\right) 3 p\left({ }^{2} P\right) 5 d$ at $52.478 \mathrm{eV},\left({ }^{1} S\right) 3 s\left({ }^{2} S\right) 3 p$ at $52.614 \mathrm{eV}$, and $\left({ }^{1} S\right) 3 d\left({ }^{2} D\right) 3 p$ at $57.574 \mathrm{eV}$. These resonances coincide with the thresholds of satellites $11,11 \mathrm{a}$, and 5 , suggesting that satellites 11a and 5 may exist only on these resonances because of the autoionization decay. Satellite 11 is clearly observed at higher photon energies. ${ }^{12,13,32}$ The complexity of the threshold spectrum may result from the correlation between the excited electron and photoelectron in two ways: nonresonantly because both electrons are slow and resonantly from the decay of doubly excited states.

The last column of Table II presents the satellite cross sections inferred from the satellite to $2 s$ branching ratio vaues derived from the threshold scan. Figure 8 shows the satellite $\sigma$ from a series of constant kinetic energy scans from 0 to $1 \mathrm{eV}$ taken at $48^{\circ}$, near the pseudo-magicangle. For the Ne scans, the counts were first normalized to the electron current in the storage ring and then divided by a separate scan of the $I(h v)$ to ring current ratio. This procedure was necessary because of instabilities in the photomultiplier tube used to monitor the photon flux. The uncertainty of this normalization is estimated to be $\leqq 5 \%$. The light intensity is corrected for higher-order components, whose contribution varied between $8 \%$ and 23\%. Figure 8 also contains the $\sigma$ values at higher kinetic energies from Heimann et al. ${ }^{12}$ and Becker et al. ${ }^{13}$ In Fig. 8 and Table II the error bars include the uncertainty from both the normalization and the higher-order correction.

The Ne satellite intensities will be discussed in two parts. First, the cross sections at threshold will be considered, then the variation of $\sigma$ with kinetic energy. The general discussion of $\mathrm{FanO}^{33}$ and the He calculation of Green $^{30}$ [shown in Fig. 6(a)] make predictions at threshold for how the excited electron goes into the different $l$ orbitals. The distribution over $l$ should change as $n$ increases, shifting to higher $l$. Figure $6(\mathrm{~b})$ shows the observed distribution for the neon valence satellites. The $n p$ states, which are heavily favored in the shake-up picture, are strong but not dominant. For satellite final states with $n=3$, the $s$ and $p$ orbitals receive nearly equal frac- 


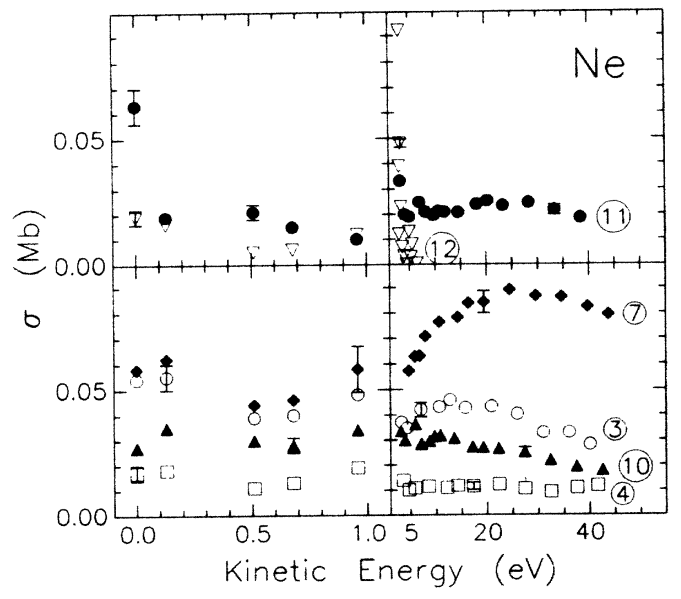

FIG. 8. Ne valence satellite cross sections at low kinetic energies $(0-1 \mathrm{eV})$ from this work along with $\sigma$ values at higher kinetic energies from Heimann et al. (Ref. 12) (satellites 4-11) and from Becker et al. (Ref. 13) (satellite 12). For each satellite typical error bars are displayed. In the notation of Table II, satellite 7 includes $6 a, 6,7$, and $7 a$, satellite 3 contains $3 a, 3$, and $3 b$, and satellite 4 includes 4 and 5 .

tions while $d$ orbitals are more infrequent. When the excited electron goes to the $n=4$ or $n=5$ shell, the fraction of $s$ orbitals is small while larger, similar portions are observed in $p$ and $d$ (or $f$ ) orbitals. Qualitatively, the Ne satellites at threshold show the expected behavior of favoring higher $l$ as $n$ increases.

It is interesting to consider the distribution among the core term symbols at threshold: the ${ }^{3} P:{ }^{1} D::^{1} S$ intensities vary as ${ }^{3} P::^{1} D:{ }^{1} S=9: 8: 0.3$. The difference between the measured values and the statistical ratios results in part from the enhancement of the ${ }^{1} D$ satellite 11 on a resonance and from the location of the ${ }^{1} S$ states at high binding energies, where some satellite peaks could not be assigned. The result at threshold contrasts with the observation at $130-150-\mathrm{eV}$ photon energy of Wuilleumier and Krause 9:(17-22):(@6). ${ }^{32}$ Their measured values may be affected by unresolved contributions in their spectra.

In the kinetic energy range from 0 to $1 \mathrm{eV}$, the intensities of satellite 11 and 12 undergo large changes. As discussed above, doubly excited states may autoionize into satellite channels enhancing the satellite intensity. In a constant kinetic energy scan, a resonantly enhanced peak shows a number of characteristic features, seen for example in satellite 11 at threshold. The width of this peak in the $0 \mathrm{eV}$ scan is $0.12 \mathrm{eV}$, less than the experimental resolution. Similarly, the $h v$ position of satellite 11 is not quite right for its binding energy. If the monochromator width is decreased to 0.09 , then the branching ratio of satellite 11 relative to the $2 s$ intensity is increased by 17(6)\%.

For satellite 11 the enhancement at threshold by a factor of 3 is caused by the resonance $\left({ }^{3} P\right) 3 p\left({ }^{2} P\right) 4 d$, which shares neither core term symbol nor excited orbital with the satellite. In contrast, a doubly excited state with a $3 s$ orbital, $\left({ }^{1} S\right) 3 s\left({ }^{2} S\right) 3 p$, gives little intensity to this satellite channel. This resonance instead strongly enhances satellite $12,{ }^{13}$ whose final state also has a $3 \mathrm{~s}$ orbital. At $0.7 \mathrm{eV}$ kinetic energy, Becker et al. ${ }^{13}$ measured a satellite 11 intensity of $0.026(2) \mathrm{Mb}$, which is somewhat larger than the present $\sigma$ result.

Satellite 12 decreases by a factor of 3 from 0 to $0.5 \mathrm{eV}$ kinetic energy. No doubly excited state has been observed $^{36}$ near the satellite 12 threshold, which suggests a continuum-state CI effect. As discussed above, the intensity of the $\mathrm{He}(2 p)$ satellite decreases with increasing kinetic energy because of continuum-state CI. However, the energy range over which satellite 12 diminishes is very much smaller than the range for $\mathrm{He}(2 p)$. At $1.0 \mathrm{eV}$ satellite 12 is seen to be increasing again because it approaches the $\left({ }^{1} D\right) 3 s\left({ }^{2} D\right) 4 p$ resonance at $50.565 \mathrm{eV}$. The mean of this line is shifted by $0.24 \mathrm{eV}$ because the autoionizing electrons are at the uppermost edge of the analyzer kinetic energy window. On the high- $h v$ side of this resonance, Becker et al. ${ }^{13}$ observed the satellite 12 intensity to be enhanced.

The satellites other than 11 and 12 are little affected by the presence of resonances. One would expect, for example, that satellite 10 would be enhanced dramatically at $0.6 \mathrm{eV}$ on the $\left({ }^{1} D\right) 3 p\left({ }^{2} P\right) 4 s$ resonance. Curiously, this resonance appears to have little effect on the other satellite channels, either. ${ }^{13}$ There seems to be something special about satellites 11 and 12, which make them more likely to be resonantly enhanced. Codling et al. ${ }^{36}$ observe the doubly excited resonances becoming weaker with increasing photon energy. Hence, most of the strong resonances are located below the thresholds of the higher satellites, and as a result coupling to satellites 11 and 12 is favored. To understand in general which doubly excited states decay into which satellite channels requires further work. At present, a weak rule might state that when a doubly excited state shares the excited orbital or core-term symbol with one or more open satellite channels, the resonance will usually couple to one of the satellite channels.

The satellites as a whole appear to become somewhat less intense at $0.5-0.7 \mathrm{eV}$. This variation could result from the multiplication by $\sigma_{2 s}$ if the $2 s$ intensity does not change linearly as assumed. Comparing the $2 s$ intensity from 1.5 to $0.1 \mathrm{eV}$ with the calculated analyzer transmission confirms that $\sigma_{2 s}$ is slowly varying and that it is enhanced by $10-20 \%$ between 0.4 and $0.7 \mathrm{eV}$. The origin of the increase is probably autoionization from doubly excited states such as $\left({ }^{1} D\right) 3 s\left({ }^{2} D\right) 3 p$ at $48.91 \mathrm{eV}$ and $\left({ }^{3} P\right) 3 s\left({ }^{2} P_{3 / 2}\right) 7 p$ at $48.98 \mathrm{eV}$. The apparent overall drop in the satellite intensities at $0.5-0.7 \mathrm{eV}$ is most likely an artifact resulting from this uncertainty in $\sigma_{2 s}$.

In comparison with higher kinetic energies, the intensities at threshold are either similar (satellites 7 and 10) or higher $(3,4,11$, and 12). This result suggests that the adiabatic limit does not exist. A recent calculation for $\mathrm{Ne}$ $2 p \rightarrow 3 p$ shake-up ${ }^{37}$ predicts that the satellite intensity at threshold is closer to the sudden limit than to the adiabatic limit. Classically, the velocity of the photoelectron as it passes through the valence orbitals is not low even at threshold. From an alternative point of view, the oscilla- 
TABLE III. Asymmetry parameters of Ne valence satellites at three kinetic energies: 0.5 and 1.0 from the present work, 5 $\mathrm{eV}$ from Ref. 12 and $\beta_{2 p}$ from Ref. 38.

\begin{tabular}{lccc}
\hline \hline Peak & $\beta(0.5 \mathrm{eV})$ & $\beta(1.0 \mathrm{eV})$ & $\beta(5 \mathrm{eV})$ \\
\hline $2 p$ & $-0.55(7)$ & $-0.63(7)$ & $-0.18(7)$ \\
$2 s$ & 2 & 2 & 2 \\
12 & $-0.9(3)$ & & \\
11 & $0.2(3)$ & $1.0(6)$ & $1.7(3)$ \\
10 & $-0.2(5)$ & $0.0(3)$ & $-0.2(1)$ \\
7 & $-0.2(2)$ & $-0.2(3)$ & \\
& & & $0.0(1)$ \\
6 & $-0.5(2)$ & $-0.2(4)$ & $0.0(1)$ \\
4 & $0.4(3)$ & & $0.4(2)$ \\
3 & $0.5(2)$ & $0.3(4)$ & \\
\hline \hline
\end{tabular}

tor strength will be a continuous function from the doubly excited Rydberg series across the corresponding satellite threshold. $^{14}$ Over the total, measured kinetic energy range, the $\mathrm{He}$ and $\mathrm{Ne}$ satellite intensities (except for the $\mathrm{Ne}$ satellite 7) are not observed to decrease as threshold is approached, contrary to the expectations of the shake-up model. ${ }^{2}$

\section{B. Neon asymmetry parameters $(\beta)$}

Table III shows the $\mathrm{Ne} \beta$ values measured at 0.5 and $1.0 \mathrm{eV}$ kinetic energies. The asymmetry parameters were calculated from intensities at $0^{\circ}$ and $48^{\circ}$ with the analyzer efficiency calibrated with the Ne $2 s$ main line. The error bars include systematic errors from the calibration. While the uncertainties are large, some qualitative observations can be made. Satellite 12 is seen to have $\beta=-1$ within error. For satellite 12 there is only one possible photoelectron wave with $l=1$. According to angular momentum transfer theory ${ }^{39}$ this transition is parity unfavored and must have a constant $\beta=-1$. The only dramatic $\beta$ variation occurs for satellite 11 . While the low $\beta$ value at $0.5 \mathrm{eV}$ might result from the $\left({ }^{1} S\right) 3 s\left({ }^{2} S\right) 3 p$ resonance at $52.614 \mathrm{eV},{ }^{36}$ no corresponding effect is observed in the $\sigma$ of Fig. 8. Near threshold, satellites 7 and 10 have $\beta$ like the $2 p$ main line, which has been observed at higher kinetic energies ${ }^{12}$ and is expected because of their ${ }^{2} P^{\circ}$ final state. Lastly, for satellite 3 the nearly constant $\beta$ at higher kinetic energies ${ }^{12}$ continues close to threshold.

The satellites of both $\mathrm{He}$ and Ne have small or negative $\beta$ values near threshold. In the case of $\mathrm{Ne}$, one must hesi- tate to infer an angular correlation between the excited and ejected electrons because the main line $\beta_{2 p}$ is also negative. The $\mathrm{Ne} \beta$ results show one satellite to originate from a parity unfavored transition. For two ${ }^{2} P^{\circ}$ satellites (7 and 10) $\beta$ is similar to $\beta_{2 p}$ near threshold as well as at higher kinetic energies.

\section{CONCLUSION}

We have directly measured for the first time to our knowledge, threshold intensities of photoelectron satellite lines. In He correlation satellites up to $n=8$ were observed. The angular-distribution asymmetry parameter $\beta$ of these satellites becomes increasingly negative with higher principal quantum number $n$ of the excited electron. This result agrees with the predictions of a theoretical treatment of the long-range dipole interaction. In $\mathrm{Ne}$ the threshold spectrum contains lines with high spin and orbital momentum and is more complex than the spectra obtained at higher photon energies. The qualitative prediction of an approach toward the adiabatic limit with satellite intensities decreasing toward threshold is not seen (except for the Ne satellite 7). Near threshold the He $1 s$ and $\mathrm{Ne} 2 p$ satellite intensities vary slowly except at resonances, where some satellites show rapid enhancement. Further theoretical and experimental work toward understanding the selective decay of doubly-excited states into satellite channels will be valuable.

\section{ACKNOWLEDGMENTS}

We would like to thank Professor B. Sonntag, H. E. Wetzel, and M. Richter for their assistance with the beam line and for their interest. We would also like to thank C. Greene for helpful discussions. This work was supported in part by the Director, Office of Energy Research, Office of Basic Energy Sciences, Chemical Sciences Division of the U.S. Department of Energy under Contract No. DEAC03-76SF00098, and in part by the Bundesminister für Forschung und Technologie (Bonn, Federal Republic of Germany). It was mainly performed at the Hamburger Synchrotronstrahlungslabor at Deutsches ElektronenSynchrotron (DESY). Some preliminary work was done at the Stanford Synchrotron Radiation Laboratory, which is supported by the U.S. Department of Energy's Office of Basic Energy Sciences. One of us (P.A.H.) acknowledges support from the Sonderforschungsbereich 161 at Freie Universität Berlin which is supported by the Deutsche Forschungsgemeinschaft.

${ }^{8}$ D. W. Lindle, T. A. Ferrett, U. Becker, P. H. Kobrin, C. M. Truesdale, H. G. Kerkhoff, and D. A. Shirley, Phys. Rev. A 31, 714 (1985), and references therein.

${ }^{9}$ V. Schmidt, H. Derenbach, and R. Malutzki, J. Phys. B 15 , L523 (1982).

10V. L. Jacobs and P. G. Burke, J. Phys. B 5, L67 (1972); K. A. Berrington, P. G. Burke, W. C. Fon, and K. T. Taylor, ibid. 15, L603 (1982); P. Scott and P. G. Burke, ibid. 17, 1321 (1984).

${ }^{11}$ G. B. Armen, T. Åberg, K. R. Karim, J. C. Levin, B. Crasemann, G. S. Brown, M. H. Chen, and G. E. Ice, Phys. 
Rev. Lett. 54, 182 (1985).

12P. A. Heimann, C. M. Truesdale, H. G. Kerkhoff, D. W. Lindle, T. A. Ferrett, C. C. Bahr, W. D. Brewer, U. Becker, and D. A. Shirley, Phys. Rev. A 31, 2260 (1985).

${ }^{13}$ U. Becker, R. Hölzel, H. G. Kerkhoff, B. Langer, D. Szostak, and R. Wehlitz, Phys. Rev. Lett. 56, 1120 (1986).

${ }^{14}$ M. Y. Adam, P. Morin, and G. Wendin, Phys. Rev. A 31, 1426 (1985).

${ }^{15}$ A. Fahlman, M. O. Krause, and T. A. Carlson, J. Phys. B 17, L217 (1984); A. Fahlman, M. O. Krause, T. A. Carlson, and A. Svensson, Phys. Rev. A 30, 812 (1984).

${ }^{16}$ T. Baer, P. M. Guyon, I. Nenner, A. Tabche-Fouhaille, R. Botter, L. F. A. Ferreira, and T. R. Govers, J. Chem. Phys. 70, 1585 (1979).

${ }^{17}$ R. Stockbauer, Int. J. Mass Spectrom. Ion Phys. 25, 89 (1977).

${ }^{18}$ S. Cvejanovic and F. H. Read, J. Phys. B 7, 1841 (1974).

${ }^{19}$ M. G. White, R. A. Rosenberg, G. Gabor, E. D. Poliakoff, G. Thorton, S. H. Southworth, and D. A. Shirley, Rev. Sci. Instrum. 50, 1268 (1979).

${ }^{20}$ W. C. Wiley and I. H. McLaren, Rev. Sci. Instrum. 26, 1150 (1955).

${ }^{21}$ F. Wuilleumier and M. O. Krause, J. Electron Spectrosc. Relat. Phenom. 15, 15 (1979); J. A. R. Samson and J. L. Gardner, Phys. Rev. Lett. 33, 671 (1974).

${ }^{22}$ U. Becker, R. Hölzel, H. G. Kerkhoff, B. Langer, D. Szostak, and $\mathbf{R}$. Wehlitz (unpublished).

${ }^{23}$ C. N. Yang, Phys. Rev. 74, 764 (1948).
${ }^{24}$ G. V. Marr and J. B. West, At. Data Nucl. Data Tables 18, 497 (1976).

${ }^{25}$ U. Fano and J. W. Cooper, Phys. Rev. 137, A1364 (1965).

${ }^{26}$ D. W. Lindle, P. A. Heimann, T. A. Ferrett, and D. A. Shirley (unpublished).

${ }^{27}$ C. Greene, Phys. Rev. Lett. 44, 869 (1980).

${ }^{28}$ D. R. Herrick, Phys. Rev. A 12, 413 (1975); O. Sinanoglu and D. R. Herrick, J. Chem. Phys. 62, 886 (1975).

${ }^{29}$ J. M. Bizau, F. Wuilleumier, P. Dhez, D. L. Ederer, T. N. Chang, S. Krummacher, and V. Schmidt, Phys. Rev. Lett. 48, 588 (1982).

${ }^{30} \mathrm{C}$. Greene (private communication).

${ }^{31}$ W. Persson, Phys. Scr. 3, 133 (1971)

${ }^{32}$ F. Wuilleumier and M. O. Krause, Phys. Rev. A 10, 242 (1974).

${ }^{33}$ U. Fano, J. Phys. B 7, L401 (1974).

${ }^{34}$ G. H. Wannier, Phys. Rev. 90, 817 (1953).

${ }^{35}$ G. B. Armen, T. Åberg, J. C. Levin, B. Crasemann, M. H. Chen, G. E. Ice, and G. S. Brown, Phys. Rev. Lett. 54, 1142 (1985).

${ }^{36}$ K. Codling, R. P. Madden, and D. L. Ederer, Phys. Rev. 155, 26 (1967).

${ }^{37}$ T. D. Thomas, J. Electron Spectrosc. Relat. Phenom. 40, 259 (1986).

${ }^{38}$ S. H. Southworth, A. C. Parr, J. E. Hardis, J. L. Dehmer, and D. M. P. Holland, Nucl. Instrum. Methods A246, 782 (1986).

${ }^{39}$ D. Dill and U. Fano, Phys. Rev. Lett. 29, 1203 (1972). 